\title{
Avaliação da separação acetona-água em uma coluna de recheio
}

\author{
Evaluation of acetone-water separation in a packing column \\ Evaluación de la separación de acetona-agua en una columna de relleno
}

Recebido: 22/07/2021 | Revisado: 28/07/2021 | Aceito: 30/07/2021 | Publicado: 05/08/2021

\author{
João Carlos Ferreira Lima \\ ORCID: https://orcid.org/0000-0003-0956-9300 \\ Universidade Federal da Bahia, Brasil \\ E-mail: joaocarlos.jcfl@hotmail.com \\ Jarlon Conceição da Costa \\ ORCID: https://orcid.org/0000-0002-0679-488X \\ Universidade Federal da Bahia, Brasil \\ E-mail: Jharlon@hotmail.com \\ Silvana Mattedi \\ ORCID: https://orcid.org/0000-0003-4816-7494 \\ Universidade Federal da Bahia, Brasil \\ E-mail: silvana@ufba.br \\ Luiz Mário Nelson de Góis \\ ORCID: https://orcid.org/0000-0001-8536-8936 \\ Universidade Salvador, Brasi \\ E-mail: Luiz.gois@unifacs.br
}

\begin{abstract}
Resumo
No presente trabalho avalia-se a operação de uma torre de recheio com anéis de vidro no processo da absorção da acetona pela água. O estudo é baseado em dados experimentais de Queda de Pressão e dos níveis de concentração coletados na saída do aparelho. Influências dos parâmetros de operação e de geometria são avaliadas através do método ANOVA e modelos empíricos tanto para concentrações de saída como para queda de pressão são também apresentados.
\end{abstract}

Palavras-chave: Queda de pressão; Coluna recheada; Absorção.

\begin{abstract}
In the present $n$ the present work, the operation of a filling tower with glass rings in the process of acetone absorption by water is evaluated. The study is based on experimental data of Pressure Drop and concentration levels collected at the outlet of the device. Influences of operating parameters and geometry are evaluated using the ANOVA method and empirical models for both outlet concentrations and pressure drop are also presented.

Keywords: Pressure drop; Packed columns; Absorption.
\end{abstract}

\section{Resumen}

En el presente trabajo se evalúa el funcionamiento de una torre de llenado con anillos de vidrio en proceso de absorción de acetona por agua. El estudio se basa en datos experimentales de caída de presión y niveles de concentración recopilados en la salida del dispositivo. Las influencias de los parámetros operativos y la geometría se evalúan utilizando el método ANOVA y también se presentan modelos empíricos tanto para las concentraciones de salida como para la caída de presión.

Palabras clave: Caída de presión; Columnas de relleno; Absorción.

\section{Introdução}

A absorção é uma operação em que uma mistura gasosa é contatada com um líquido com o objetivo de separar preferencialmente um ou mais componentes A operação requer então a transferência de massa do soluto da corrente gasosa para a corrente líquida. Quando a transferência ocorre na direção oposta, ou seja, da fase líquida para a fase gasosa, é chamada de dessorção ou "stripping" (Treybal, 1980).

A operação ocorre em torres de recheio ou colunas recheadas, onde vários fatores influenciam na taxa de transferência de massa que acontece entre as duas fases da operação. Estes fatores vão desde o tipo de recheio utilizado até 
parâmetros operacionais como, vazões, temperatura, concentrações, etc.

$\mathrm{Na}$ absorção existe sempre a presença de duas fases, a contínua e a dispersa, onde um soluto presente na fase dispersa, é transportado para a fase contínua através do contato em contracorrente. Daí dados como área interfacial de contato, coeficiente de transferência de massa, fração de holdup, diâmetro de gotas, dentre outros, se tornam de fundamental importância na operação das absorvedoras já que estão diretamente ligadas ao transporte de massa que ocorre no interior das torres.

O comportamento fluidodinâmico, ou a fluidodinâmica das absorvedoras surge como o estudo da influência dos parâmetros de operação com o objetivo da busca de melhor eficiência das torres. Nessa linha, a determinação da queda de pressão que ocorre na torre ou o conhecimento dos níveis de influência dos parâmetros operacionais e geométricos surgem como de grande importância para o bom funcionamento das absorvedoras.

Portanto, no presente trabalho é apresentado um estudo avaliativo de uma absorvedora que opera com o sistema ar atmosférico-acetona-água e obtidos dados de queda de pressão e da concentração de soluto absorvida pela água. Uma avaliação dos níveis de influências dos parâmetros, operacionais e geométricos, utilizando o método ANOVA é apresentado, além de duas equações empíricas para queda de pressão e concentração de acetona absorvida pela água.

\subsection{Análise da literatura}

Vários trabalhos publicados na literatura têm dedicado atenção ao estudo da operação das absorvedoras. Os estudos geralmente tratam de dados relacionados com características dos recheios, a transferência de massa ou da queda de pressão existente na coluna. Outros trabalhos estão relacionados, por exemplo com simulações envolvendo comparações de modelos utilizados para a eficiência da separação nas colunas.

Dentre os parâmetros operacionais relacionados a queda de pressão, segundo Heyouni et al. (2002) pode ser considerado como o parâmetro mais importante no critério de configuração para uma torre de recheio, já que quantifica a dispersão das fases e é sendo decisivo na eficiência da operação. Sua dependência se resume em três fatores: a velocidade das fases líquida e gasosa, do tipo do fluído e do tipo de recheio envolvido, esses fatores são estudados também de Bernardes \& Góis (2020) e Lima et al. (2021). E aí, como o escoamento descendente do líquido ocupa os mesmos canais que o escoamento ascendente de gás ou líquido, a queda de pressão é sempre uma função das duas vazões, de líquido e de gás.

Segundo Caldas et al. (2003) vários são os modelos existentes na literatura que podem ser aplicados na avaliação da perda de carga $(\Delta \mathrm{P} / \mathrm{z})$, nas colunas de recheio. Nos modelos geralmente são tratadas as colunas com leitos secos, onde somente a fase gasosa está presente, e as com leitos molhados, onde as duas fases, líquida e gasosa estão presentes. No caso das operações com leitos secos, segundo Caldas (2003) destacam-se como principais modelos, o de Ergun (1952) e o de Treybal (1980. Enquanto que para as colunas com leitos molhados, ou com as duas fases presentes na operação, os métodos de Leva (1953), Niranjan (1983), Billet (1984), Graff (1985) e o de Prahl (1970).

Segundo Botta (2015) existem também métodos gráficos para a determinação da queda de pressão nas absorvedoras, que apresentam respostas bastante satisfatórias, o que também foi estudado na pesquisa de Lima et al. (2021). Estes métodos envolvem gráficos de Correlação Generalizada para a Queda de Pressão (CGQP) envolvendo o escoamento bifásico e situados na região dinâmica da coluna.

Já trabalhos como o publicado por Nasser (2009) dentre outros tratam de avaliações de desempenho de absorvedoras envolvendo simulações e análises estatísticas, ou comparações com outros processos de separação. 


\section{Metodologia}

Essa investigação foi conduzida através de uma pesquisa exploratória, dentro de uma abordagem quali-quantitativa, uma vez que se utilizou de coleta de dados por meio do uso de medições de grandezas, bem como envolveu interpretações da fenomenologia sistêmicas e das respostas obtidas (Cajueiro, 2013; Pereira, et al., 2018; Koche 2011).

Os testes experimentais foram conduzidos em uma coluna de absorção de acrílico com diâmetro de $0,07 \mathrm{~m}$ e comprimento de $1,0 \mathrm{~m}$, com recheio de anéis de vidro com $0,0095 \mathrm{~m}$ de comprimento totalizando uma altura total de recheio $0,79 \mathrm{~m}$. Na Figura 3 é mostrada uma fotografia da coluna utilizada.

Figura 1: Fotografia da coluna utilizada nos experimentos.

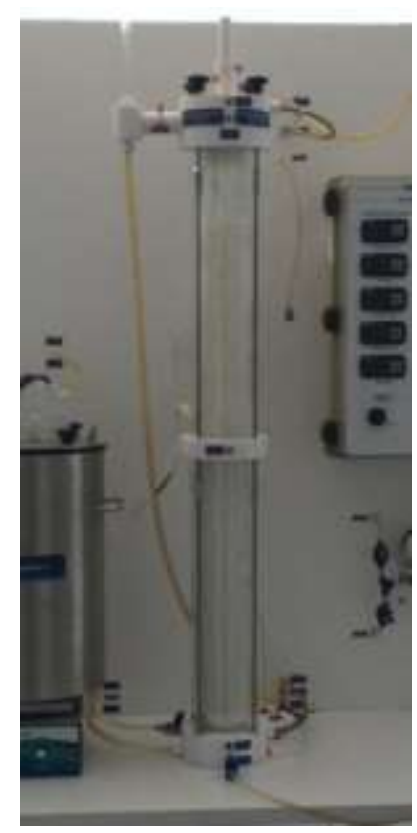

Fonte: Autores.

Nos testes as alimentações da coluna foram feitas pela base (fase gasosa) e pelo topo (fase líquida), sendo ambas controladas por rotâmetros. Já a queda de pressão era obtida através de um manômetro em U instalado nos dois extremos da torre. Conforme mostrado no fluxograma da Figura 2 a alimentação gasosa era composta de uma mistura formada pelo ar atmosférico e acetona. Primeiramente o ar atmosférico proveniente do compressor, passava por um leito contendo sílica, para a redução da umidade e depois era enviado a um saturador que continha acetona. A mistura formada, ar-acetona, era então alimentada pela base da absorvedora, percorrendo todo o comprimento da coluna. Já a alimentação líquida era uma corrente de água pura introduzida pelo topo da coluna, também controlada por rotâmetro. Amostras de líquido foram coletadas através de seringas introduzidas na base da coluna, com auxílio de um cronômetro a qual no tempo correto eram feitas as coletas, assim e analisadas através da espectrofotometria. 
Figura 2: Fluxograma da aparelhagem experimental utilizada.

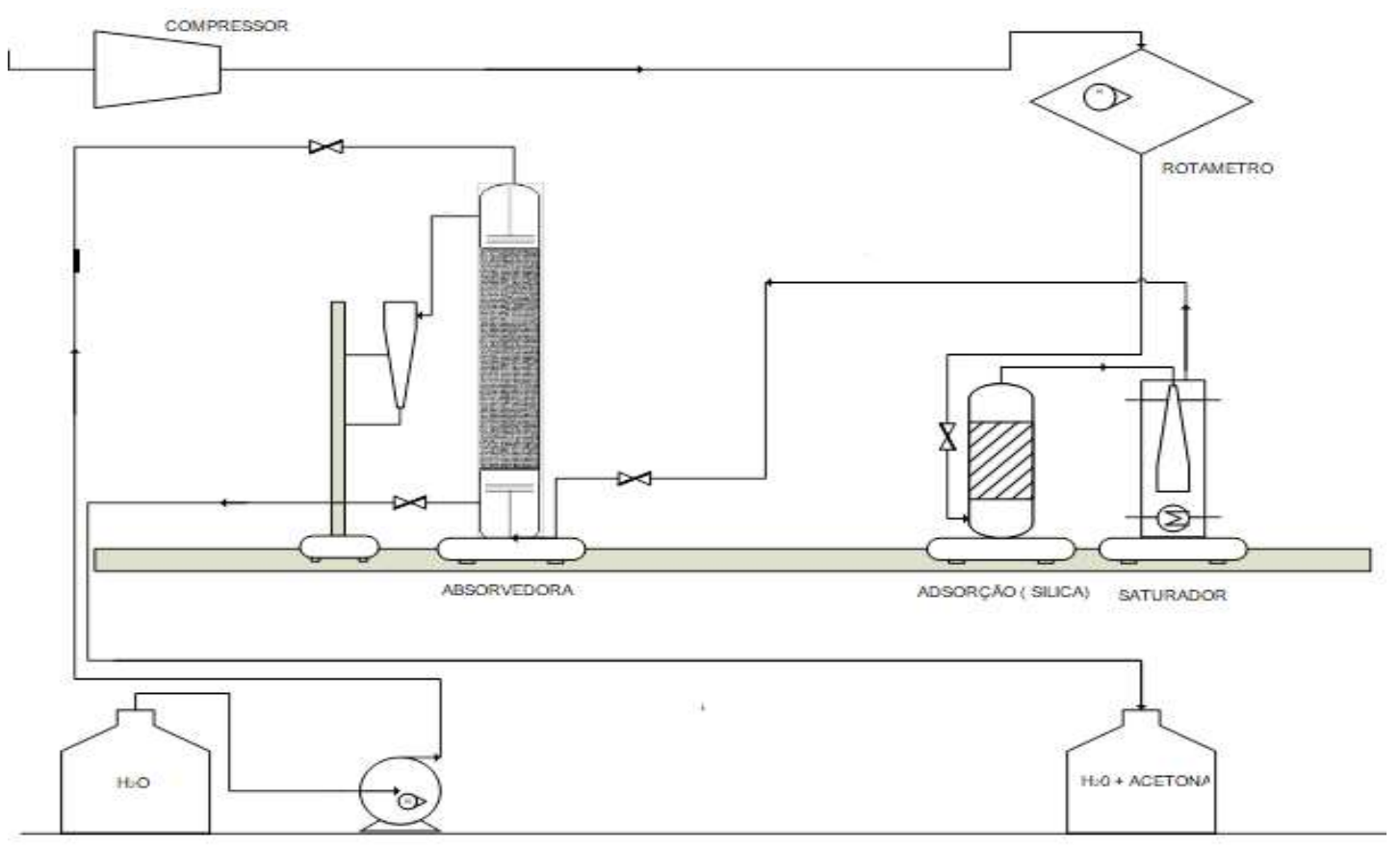

Fonte: Autores.

Na Figura 3 mostra uma fotografia de toda a unidade experimental utilizada.

Figura 3: Fotografia da montagem experimental.

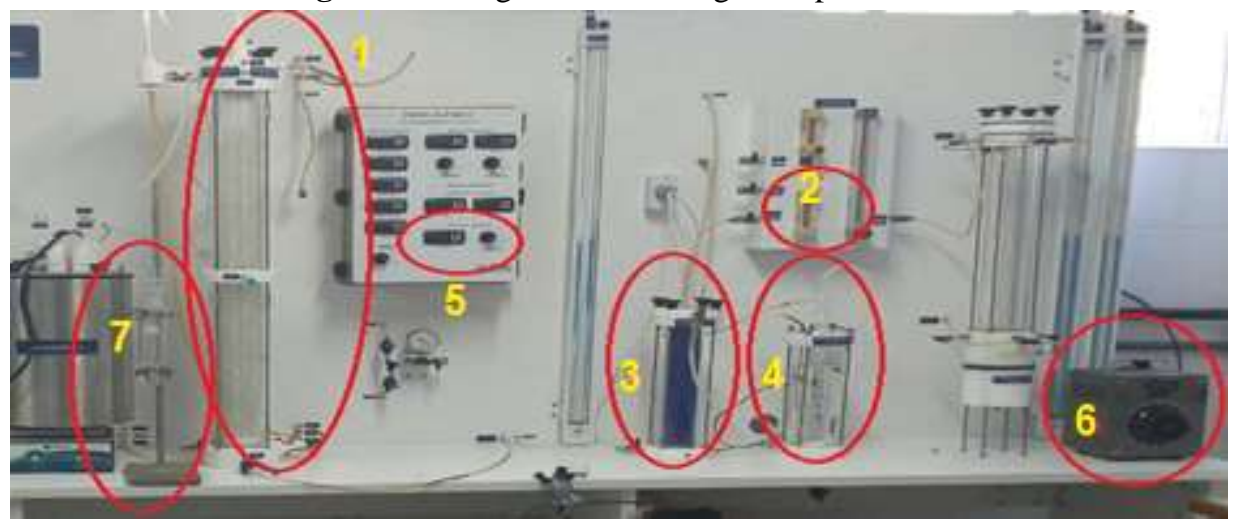

(1) Coluna de absorção, (2) Rotâmetro (3) Adsorvedora de sílica, (4) Saturador, (5) Painel de Temperatura, (6) Transformador de voltagem e (7) Borbulhador Fonte: Autores.

A leitura das concentrações de acetona nas amostras da fase aquosa obtidas na base da coluna foi feita através de espectrofotômetro UV-VIS-NIR UV-3600 Plus da SHIMADZU através da análise UV, com um comprimento de onda de $272 \mathrm{~nm}$, valor esse obtido através de uma varredura realizada em diferentes concentrações de acetona em água. Deve-se salientar que esta faixa de comprimento ficou bem próxima da utilizada por autores como, Amatuzi (1999), que também realizou medidas de concentrações de acetona em testes com água. 
Uma curva de calibração foi construída através das medidas de absorbâncias encontradas para concentrações de acetona variando entre 0,2 e 2,0 g/g, conforme mostrado na Figura 4, onde foi obtido um coeficiente de correlação $\left(\mathrm{R}^{2}\right)=$ 0,9985 .

Figura 4: Curva de calibração obtida.

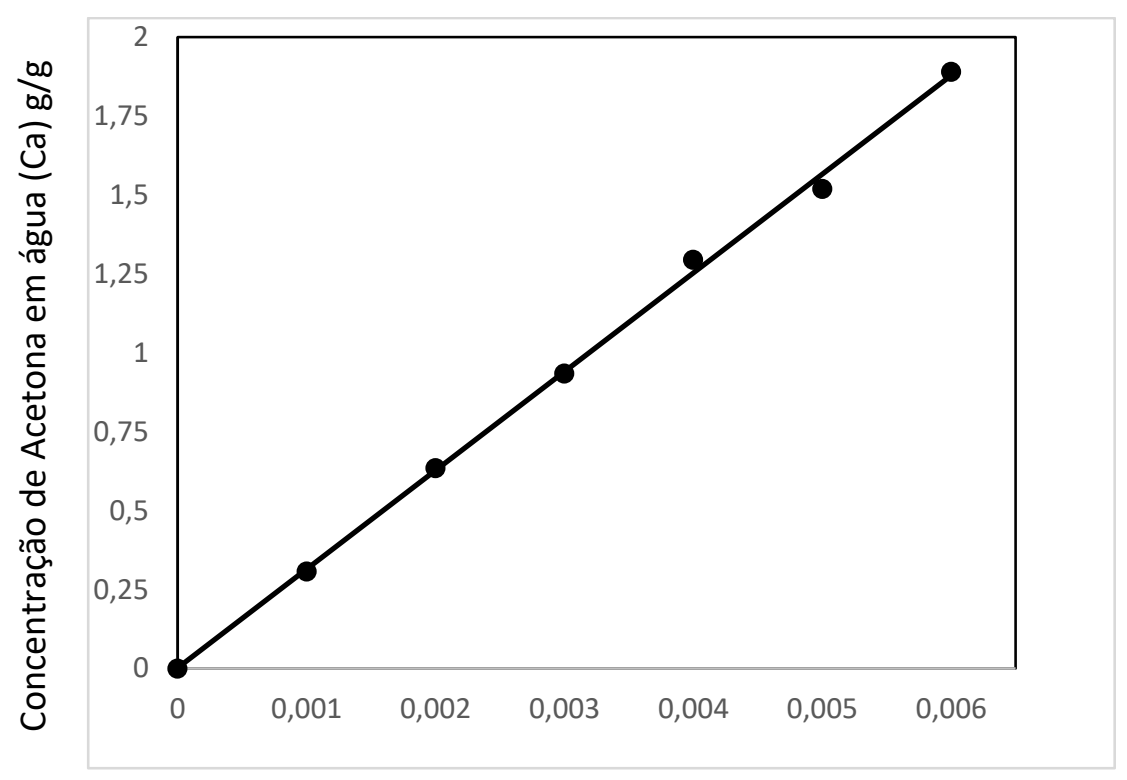

Absorbância (A)

Fonte: Autores.

A Equação 1 foi obtida para a conversão da absorbância em concentração, sendo ela a seguinte

$$
\mathrm{A}=312,71(\mathrm{Ca})+0,0021
$$

Onde:

A corresponde ao valor da absorbância e Ca ao valor da concentração em acetona (g)/água (g).

Os testes foram realizados baseados em um planejamento experimental 24 com pontos de repetição envolvendo 4 variáveis independentes que gerou um total de 19 experimentos, sendo acrescentado no estudo os pontos centrais das variáveis. As variáveis independentes foram as duas vazões de alimentação, fase dispersa e fase contínua, a temperatura, e o tempo gasto do ar no saturador de acetona. Enquanto que os parâmetros dependentes foram as concentrações de acetona na saída na líquida. As vazões de alimentação escolhidas foram de 2,0; 3,0 e 4,0 L/min para a fase dispersa e 1,13; 1,27 e 1,41 L/min para a fase contínua. Enquanto o tempo gasto do ar no saturador foram os de 1,0; 5,5 e 10 min e a temperatura da coluna variou entre $298 \mathrm{~K}$ e $335 \mathrm{~K}$. Deve-se salientar que os valores das vazões de alimentação escolhidos para os experimentos seguiram a indicação de um estudo preliminar, (Lima et al., 2021), onde foram determinadas as faixas de inundação da absorvedora. 


\section{Resultados e Discussão}

Os resultados obtidos no presente trabalho constaram primeiramente da avaliação dos níveis de importância dos parâmetros de operação da coluna, utilizando-se o tratamento estatístico. Em seguida foi obtido também uma equação empírica para a os níveis de concentração de acetona absorvida pela água. Além destes, foi encontrado também um modelo empírico envolvendo a Queda de Pressão, desenvolvido através da técnica da análise dimensional.

\subsection{Tratamento Estatístico}

O tratamento estatístico constou da avaliação dos efeitos das variáveis independentes na concentração de acetona absorção da acetona pela corrente aquosa, medida através da concentração da acetona na base da absorvedora, nos 19 ensaios realizados. A Tabela 1, mostrada a seguir, ilustra todo o planejamento experimental realizado, bem como os valores obtidos para as concentrações acetona na saída da coluna. Nas colunas da Tabela 1 são mostrados os níveis reais de Temperatura (T), Vazões das fases dispersa (Qd) e contínua (Qc), tempo de saturação (t) e as concentrações de saída de acetona (Ca). Para cada parâmetro foram codificados dois níveis, o inferior (-1) e o superior (+1).

Tabela 1: Planejamento Experimental:

\begin{tabular}{|c|c|c|c|c|c|c|c|c|c|}
\hline \multirow[b]{2}{*}{ Ensaio } & \multicolumn{2}{|c|}{$\mathrm{T}\left({ }^{\circ} \mathrm{C}\right)$} & \multicolumn{2}{|c|}{$\mathrm{Qc}(\mathrm{L} / \mathrm{min})$} & \multicolumn{2}{|c|}{$\mathrm{Qd}(\mathrm{L} / \mathrm{min})$} & \multicolumn{2}{|c|}{$t(\min )$} & \multirow{2}{*}{$\frac{\mathrm{Ca}(\mathrm{g} / \mathrm{g}) \times 10^{3}}{\text { Real }}$} \\
\hline & Código & Real & Código & real & código & Real & código & real & \\
\hline 1 & -1 & 25 & -1 & 1,13 & -1 & 2 & -1 & 1 & 2,74 \\
\hline 2 & 1 & 62 & -1 & 1,13 & -1 & 2 & -1 & 1 & 1,71 \\
\hline 3 & -1 & 25 & 1 & 1,41 & -1 & 2 & -1 & 1 & 3,18 \\
\hline 4 & 1 & 62 & 1 & 1,41 & -1 & 2 & -1 & 1 & 3,14 \\
\hline 5 & -1 & 25 & -1 & 1,13 & 1 & 4 & -1 & 1 & 3,39 \\
\hline 6 & 1 & 62 & -1 & 1,13 & 1 & 4 & -1 & 1 & 3,54 \\
\hline 7 & -1 & 25 & 1 & 1,41 & 1 & 4 & -1 & 1 & 3,91 \\
\hline 8 & 1 & 62 & 1 & 1,41 & 1 & 4 & -1 & 1 & 5,89 \\
\hline 9 & -1 & 25 & -1 & 1,13 & -1 & 2 & 1 & 10 & 2,79 \\
\hline 10 & 1 & 62 & -1 & 1,13 & -1 & 2 & 1 & 10 & 1,84 \\
\hline 11 & -1 & 25 & 1 & 1,41 & -1 & 2 & 1 & 10 & 3,71 \\
\hline 12 & 1 & 62 & 1 & 1,41 & -1 & 2 & 1 & 10 & 2,98 \\
\hline 13 & -1 & 25 & -1 & 1,13 & 1 & 4 & 1 & 10 & 3,69 \\
\hline 14 & 1 & 62 & -1 & 1,13 & 1 & 4 & 1 & 10 & 3,5 \\
\hline 15 & -1 & 25 & 1 & 1,41 & 1 & 4 & 1 & 10 & 4,68 \\
\hline 16 & 1 & 62 & 1 & 1,41 & 1 & 4 & 1 & 10 & 3,84 \\
\hline 17 & 0 & 43,5 & 0 & 1,27 & 0 & 3 & 0 & 4,5 & 3,09 \\
\hline 18 & 0 & 43,5 & 0 & 1,27 & 0 & 3 & 0 & 4,5 & 3,1 \\
\hline 19 & 0 & 43,5 & 0 & 1,27 & 0 & 3 & 0 & 4,5 & 3,43 \\
\hline
\end{tabular}

Fonte: Autores.

Com os dados do planejamento experimental foi possível construir o Diagrama de Pareto (Figura 5) e se observar os valores de "p" obtidas tanto para cada variável independente, como também para as interações entre elas. 
Figura 5: Gráfico de Pareto para as variáveis independentes e as interações de concentração de acetona em água na saída da coluna.

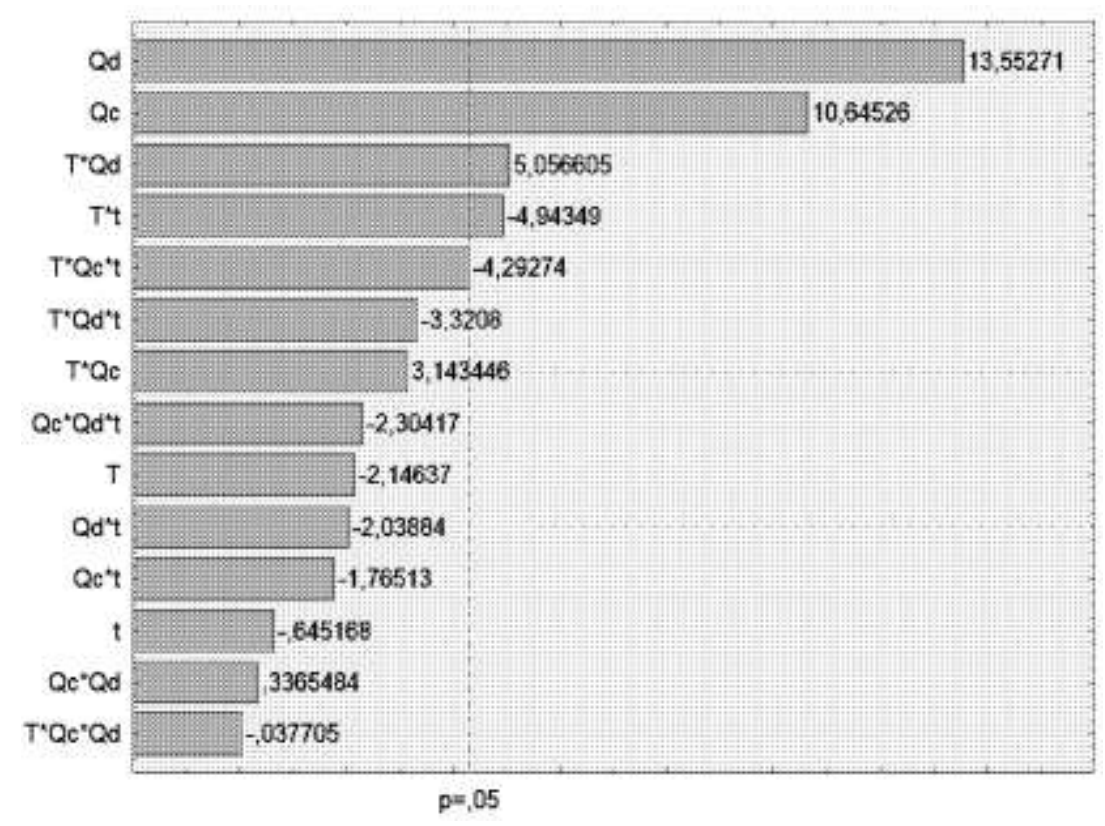

Fonte: Autores.

De acordo com Troendle (2008), a ANOVA ou análise de variância é um método para testar a igualdade de três ou mais médias populacionais, baseado na análise das variâncias amostrais. O valor do parâmetro "p" indicado na Figura 5 relaciona a probabilidade ser algo puramente ao acaso. Ou seja, se o valor "p" estiver abaixo de 0,05 é bastante provável que qualquer diferença entre os grupos é uma diferença real e não algo que aconteceu ao acaso, sendo que esse valor de 0,05 significa que existe apenas $5 \%$ de chance de seu resultado ser uma casualidade. Valores menores de "p" significam maior confiabilidade em seu teste. Esses efeitos estatisticamente são considerados significativos e os demais efeitos apresentando valores de $\mathrm{p}>0,05$, são considerados como não significativos. Essa afirmativa é inclusive também descrita por Dharma et al. (2016), a qual relata que o nível descritivo, ou probabilidade de significância (p), indica a probabilidade de erro e significância dos coeficientes de regressão, ou seja, o valor p avalia a significância estatística do modelo em relação aos dados experimentais.

Logo, analisando os dados apresentados na Tabela 2, considerando os efeitos significativos, ou seja, valores de “p” maiores que 0,05, tem-se em destaque a vazão da fase continua (Qc), a vazão da fase dispersa (Qd) e os demais efeito das interações $\left(\mathrm{T}^{*} \mathrm{Qd}, \mathrm{T}^{*} \mathrm{t}\right.$ e $\left.\mathrm{T}^{*} \mathrm{Qd} \mathrm{d}^{*} \mathrm{t}\right)$. Sendo, portanto, a temperatura $(\mathrm{T})$ e o tempo $(\mathrm{t})$, não considerados como significativos quando analisados individualmente. 
Tabela 2: Valores dos coeficientes (C) e dos valores de “p” obtidos do ANOVA.

\begin{tabular}{lcc}
\hline \multicolumn{1}{c}{ Termo } & $\mathrm{C} \times 10^{3}$ & $\mathrm{p} \times 10^{2}$ \\
\hline Constante & 3,377 & 0,0168 \\
$\underline{\mathrm{Qc}}$ & $\underline{5,080}$ & $\underline{0,8709}$ \\
$\underline{\mathrm{Qd}}$ & $\underline{6,470}$ & $\underline{0,5400}$ \\
$\mathrm{~T}$ & $-0,102$ & 16,4963 \\
$\mathrm{~T}$ & $-0,031$ & 58,4948 \\
$\mathrm{~T} * \mathrm{Qc}$ & 0,150 & 8,8042 \\
$\underline{\mathrm{T} * \mathrm{Qd}}$ & $\underline{0,241}$ & $\underline{3,6955}$ \\
$\underline{\mathrm{T} * \mathrm{t}}$ & $\underline{-0,236}$ & $\underline{3,8568}$ \\
$\mathrm{Qc} * \mathrm{Qd}$ & 0,016 & 76,849 \\
$\mathrm{Qc} * \mathrm{t}$ & $-0,084$ & 21,9586 \\
$\mathrm{Qd} * \mathrm{t}$ & $-0,097$ & 17,8319 \\
$\mathrm{~T} * \mathrm{Qc} * \mathrm{Qd}$ & $-0,002$ & 97,3348 \\
$\underline{\mathrm{T} * \mathrm{Qd}{ }^{*} \mathrm{t}}$ & $\underline{-0,205}$ & $\underline{5,0214}$ \\
$\mathrm{~T} * \mathrm{Qc} * \mathrm{t}$ & $-0,158$ & 7,9956 \\
$\mathrm{Qc} * \mathrm{Qd} * \mathrm{t}$ & $-0,110$ & 14,7725 \\
\hline
\end{tabular}

Fonte: Autores.

Ainda analisando a Tabela 2, agora destacando as interações entres os efeitos, as combinações entre a temperatura e da vazão da fase dispersa $\left(\mathrm{T}^{*} \mathrm{Qd}\right)$ e o da temperatura com o do tempo $\left(\mathrm{T}^{*} \mathrm{t}\right)$, apresentaram valores $\mathrm{p}$ também maiores que 0,05 caracterizando a significância dessas combinações. Uma observação que deve ser posta em destaque, é a da interação temperatura com a vazão da fase dispersa e o tempo ( $\mathrm{T}^{*} \mathrm{Q} \mathrm{d}^{*} \mathrm{t}$ ), pois seu valor p apresentou -se com o valor de 0,050214, ou seja, muito próximo ao valor limite para ser considerado significativo. Justamente por esse motivo, e conhecendo o sistema operacional, entende-se a sua importância, mesmo apresentando seu coeficiente com comportamento de efeito negativo no valor de $-0,205 \times 10^{-3}$.

A partir dos valores dos coeficientes mostrados na Tabela 2, percebe-se que efeito linear da vazão da fase dispersa e da vazão da fase contínua, são os efeitos que mais tem "significância", em valores absolutos. O efeito positivo mostra que ao aumentar os valores desses fatores, do menor nível para o de maior nível, deve ocorrer um aumento no valor de concentração de acetona em água (variável resposta).

Como o objetivo do processo é a absorção de acetona em água, e a coluna forneceu algumas combinações onde apresentou coeficiente que dificultam esse processo de absorção com seus efeitos negativos. Quando se comparou as interações com combinações duplas foi possível a conclusão da interação mais influente no processo, que cabe ao efeito positivo, foi o da temperatura com a vazão da fase dispersa, com valor absoluto maior que o efeito da interação da temperatura com o tempo. 
Agora, com relação aos efeitos da variação da temperatura e do tempo, estes, quando associados, se comportaram de forma negativa, o que indica que a variação no seu valor, poderão diminuir o valor de concentração de acetona em água. Porém como se apresentou significativo pelos resultados de p valor, devem ser considerados na equação de regressão gerada.

Ainda utilizando o ANOVA, a Tabela 3 mostra agora os valores obtidos para a análise de variância e a adequabilidade do modelo elaborado a partir do Teste de Fisher (F). Essa análise permite avaliar a importância fatores comparando as médias das variáveis de resposta em diferentes níveis de fatores.

Tabela 3: Análise de variância para avaliação estatística do modelo para a determinação de concentração de acetona em água.

\begin{tabular}{cccccc}
\hline Fonte de Variação & $\begin{array}{c}\text { Soma dos quadrados } \\
\mathrm{x}\left(10^{5}\right)\end{array}$ & $\begin{array}{c}\text { Graus de } \\
\text { liberdade }\end{array}$ & Média quadrática & $\begin{array}{c}\mathrm{F} \\
\text { (calculado) }\end{array}$ & $\begin{array}{c}\mathrm{F} \\
\text { (tabelado) }\end{array}$ \\
\hline Regressão & 1,47 & 14 & $7,35763 \mathrm{E}-06$ & 41,23494 & 5,873346 \\
Resíduo & 0,00 & 4 & $1,78432 \mathrm{E}-07$ & & \\
Falta de ajuste & 0,00 & 2 & 0,000000 & & \\
Erro puro & 0,00 & 2 & 0,000000 & 3,8995 & 19 \\
Total & 1,50 & 18 & & & \\
$\mathrm{R}^{2}$ & & & 0,976 & & \\
\hline
\end{tabular}

Fonte: Autores.

De acordo com Rodrigues e Lemma (2005) e Simonelli et al. (2019), o teste de Fisher (F) define a existência da regressão linear, ou seja, a probabilidade de parâmetros analisados serem independentes. Já Barros Neto et al. (2010) afirmam que o valor de $\mathrm{F}$ calculado $(41,23494)$ deve ser de quatro a cinco vezes maior que o $\mathrm{F}$ tabelado $(5,87334)$ para que o modelo gerado seja preditivo. De acordo com os dados, observa-se que F calculado foi sete vezes maior que o $\mathrm{F}$ tabelado, confirmando a adequação do modelo. Ainda sobre os valores da ANOVA (Tabela 3), o valor do coeficiente de determinação $\left(R^{2}\right)$ do modelo de regressão foi 0,976 indicando um bom ajuste do modelo, e que apenas $2,4 \%$ das variações não podem ser explicadas.

A Equação 2, mostrada a seguir, descreve o modelo codificado gerado para o nível de confiança de $95 \%$.

$$
\mathrm{Ca}=0,00337+0,00051 \mathrm{X}_{2}+0,00065 \mathrm{X}_{3}+0,00024 \mathrm{X}_{1} \mathrm{X}_{3}-0,00023 \mathrm{X}_{1} \mathrm{X}_{4}-0,00016 \mathrm{X}_{1} \mathrm{X}_{3} \mathrm{X}_{4}
$$

Onde $\mathrm{X}_{1}$ representa a Temperatura, $\mathrm{X}_{2}$ a vazão da fase contínua, $\mathrm{X}_{3}$ a vazão da fase dispersa e $\mathrm{X}_{4} \mathrm{o}$ tempo experimental.

Substituindo-se as variáveis a equação (2) passa a ser então a seguinte

$$
\mathrm{Ca}=0,00337+0,00051 \mathrm{Qc}+0,00065 \mathrm{Qd}+0,00024 \text { T.Qd- 0,00023 T.t - 0,00016 T.Qd.t }
$$

O comparativo dos valores de concentração de acetona em água entre as respostas calculadas pela modelagem proposta e as previstas pela Equação 3 podem ser observadas na Figura 6. 
Figura 6: Comparação entre os valores a concentração de acetona em água experimentais e os calculados pela equação 3.

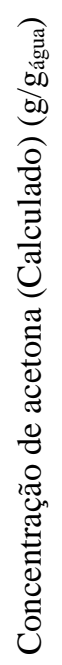

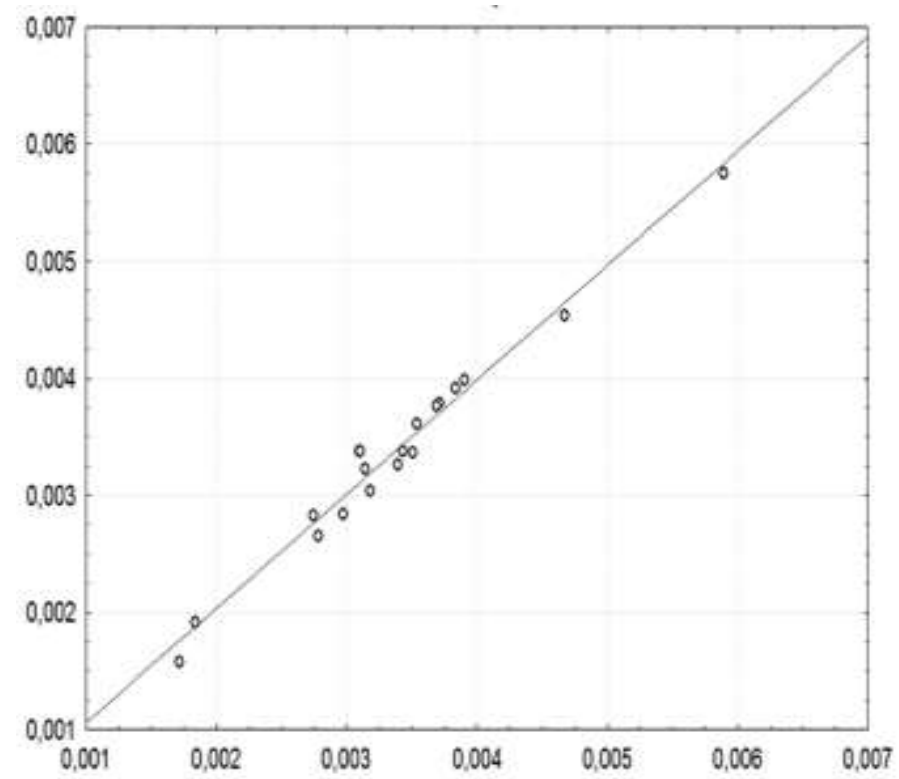

Concentração de acetona (Experimental) $\left(\mathrm{g} / \mathrm{g}_{\text {água }}\right)$ - Fonte: Autores.

\subsection{O modelo de superfície de resposta (MSR)}

Ainda sobre a influências dos parâmetros, foram plotados gráficos com base na metodologia de superfície de resposta (MSR) utilizando somente as variáveis significativas do processo. Segundo Hoffmann (2011), este tipo de gráfico permite a análise da região de máximo e de mínimo da variável resposta do sistema.

As Figuras 7 a 12 mostram as superfícies de respostas para os experimentos com as influências dos parâmetros na concentração de acetona na saída da coluna. No primeiro gráfico é mostrada a variação da concentração de acetona com a temperatura $(\mathrm{T})$ com a vazão da fase contínua $(\mathrm{Qc})$ sendo mantidos fixos a vazão da fase dispersa (Qd) e o tempo de saturação(t). Este gráfico está mostrado através da Figura 7.

Figura 7: Superfície de da variação da concentração da acetona com relação a vazão da fase contínua (Qc) e o tempo de saturação (t).

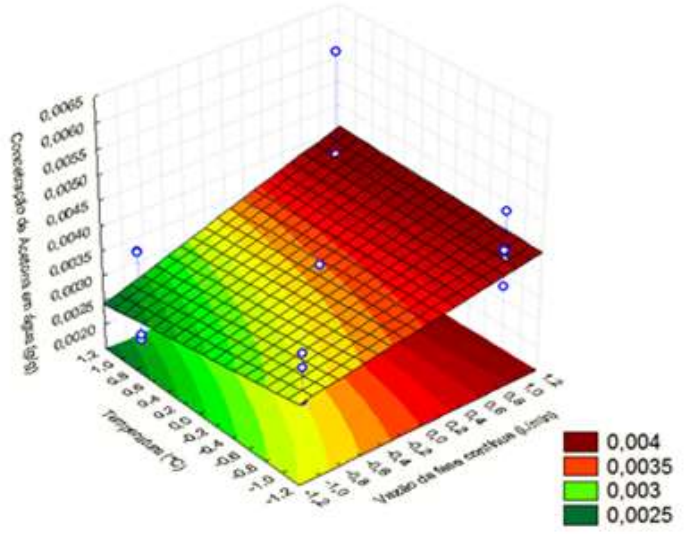

Fonte: Autores. 
No gráfico da Figura 7 verifica-se que o comportamento interativo da temperatura com a vazão da fase contínua não apresentou resultados significativos para a obtenção de maiores valores de concentração de acetona em água, já que uma pequena variação ocorreu nos valores das concentrações de acetona. Tal fato já era esperado, já que a mudança de temperatura deveria estar ligada diretamente ao saturador de ar, ou seja, vinculado a corrente de vazão da fase dispersa, e não à vazão da fase contínua.

Na Figura 8 o gráfico mostrado apresenta o comportamento interativo da vazão das duas fases, contínua e dispersa com a concentração da acetona. Neste gráfico foram mantidos fixos a temperatura $(\mathrm{T})$ e o tempo (t).

Figura 8: Superfície de resposta da variação da concentração da acetona com relação as vazões das fases contínua (Qc) e dispersa (Qd).

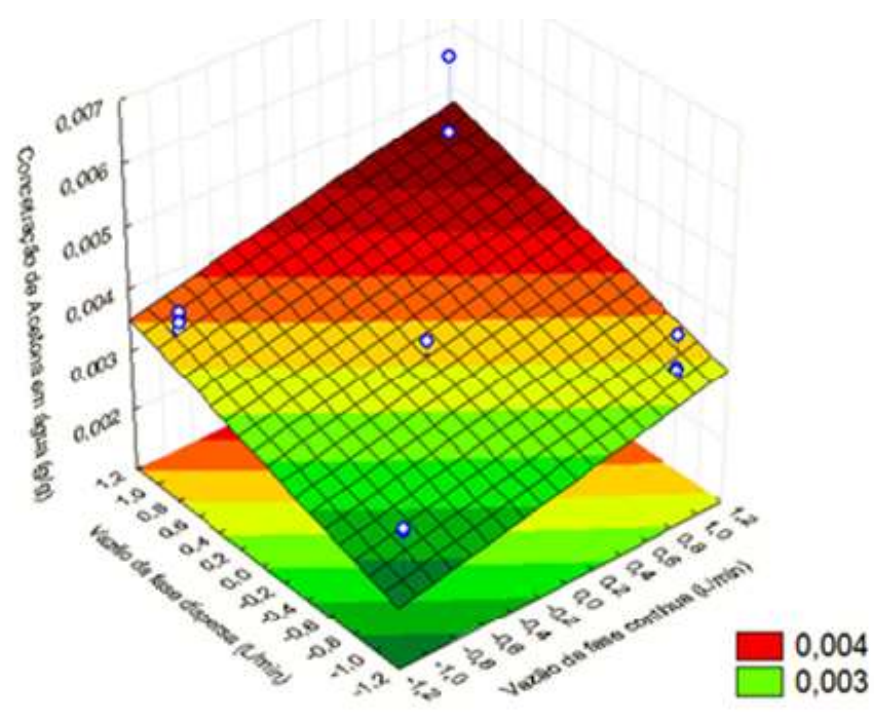

Fonte: Autores.

No gráfico da Figura 8 tem-se a interação que maior apresentou significância para a obtenção de concentração de acetona em água de modo geral. Nele identifica-se que a interação entre as vazões das fases, dispersa e contínua, apresentou uma grande variação nos valores de concentração de acetona $(0,002 \mathrm{~g} / \mathrm{g}$ para aproximadamente $0,005 \mathrm{~g} / \mathrm{g})$, o que pode ser explicado que quando as duas vazões foram testadas em seus níveis mais elevados $(+1)$, houve um maior contato das fases em contracorrente, tornando o sistema mais turbulento e aumentando a troca de massa na coluna.

Na Figura 9 é mostrada a interação da vazão da fase contínua com o tempo, com a concentração da acetona, sendo mantida fixas a vazão da fase dispersa $(\mathrm{Qd})$ e a temperatura $(\mathrm{T})$. 
Figura 9: Superfície de resposta da variação da concentração da acetona com relação as vazões das fases contínua (Qc) e o tempo $(\mathrm{t})$.

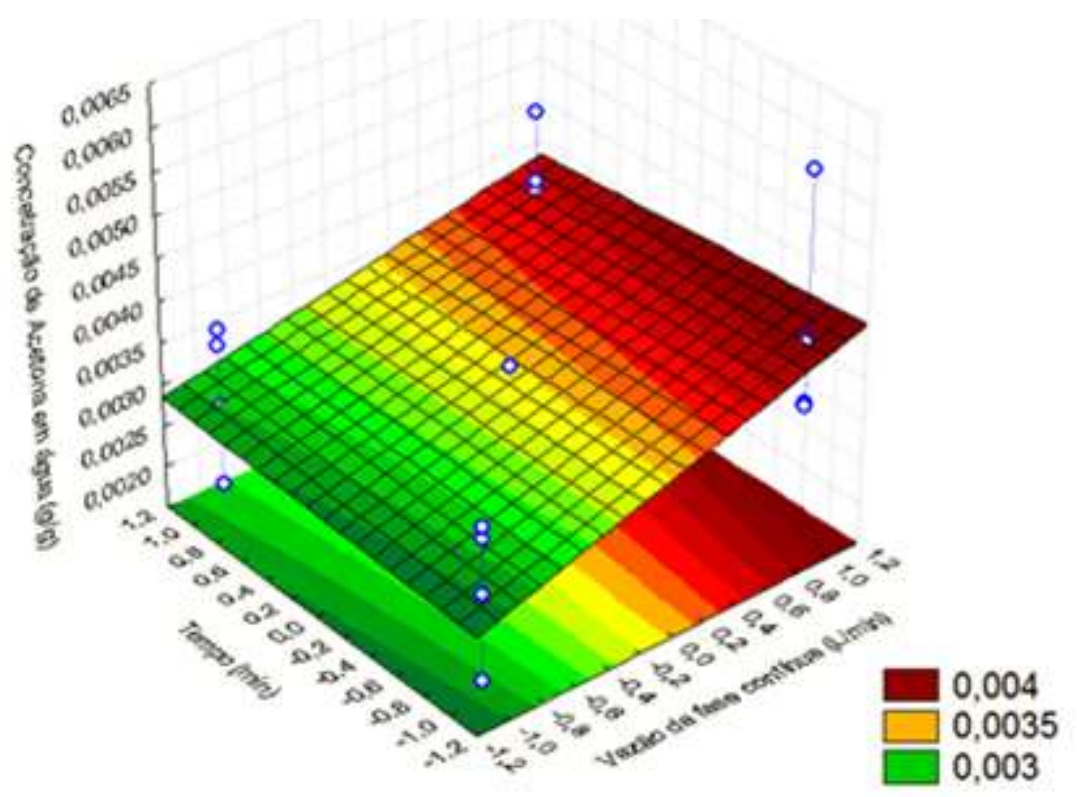

Fonte: Autores.

Neste gráfico percebe-se que o tempo exibe um caráter não tão importante no estudo, pelo que é observado, quando estudado com a vazão da fase contínua. Ou seja, a troca e massa considerando a variação do tempo de saturação (t), apresentou valores aproximadamente similares com relação a variação da concentração da acetona.

Na Figura 10 é mostrada a interação da vazão da fase dispersa com a temperatura, sendo mantidas fixas a vazão da fase continua e o tempo de saturação. 
Figura 10: Superfícies de respostas da variação da concentração da acetona com relação a vazão da fase dispersa e a temperatura.

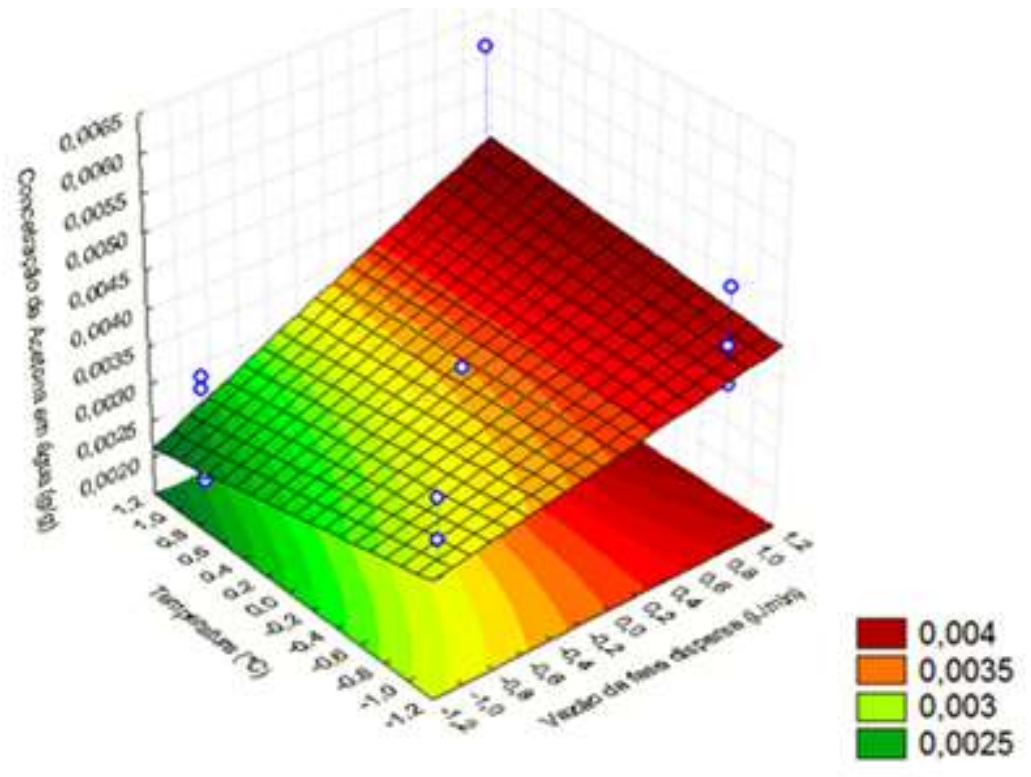

Fonte: Autores.

No gráfico da Figura 10, percebe-se que a combinação de uma maior temperatura com uma maior vazão da fase dispersa, a concentração de acetona não apresentou variação significativa com relação aos níveis inferiores. Entretanto, quando se utilizou a temperatura no maior nível (+1) com baixas vazões da fase dispersa (-1), verificou-se uma tendência de baixos valore de concentração da acetona pela água. A justificativa para o comportamento apresentado é o mesmo que foi descrito por Nasser et al. (2008). Ou seja, a absorção da acetona pela água tem um melhor comportamento quando se utiliza baixas temperaturas.

Na Figura 11 é mostrada a interação da vazão da fase dispersa e o tempo com a concentração da acetona, sendo mantidas fixas a vazão da fase continua (Qc) e o temperatura (T). 
Figura 11: Superfícies de respostas da variação da concentração da acetona com relação a vazão da fase dispersa (Qd) e o tempo $(\mathrm{t})$.

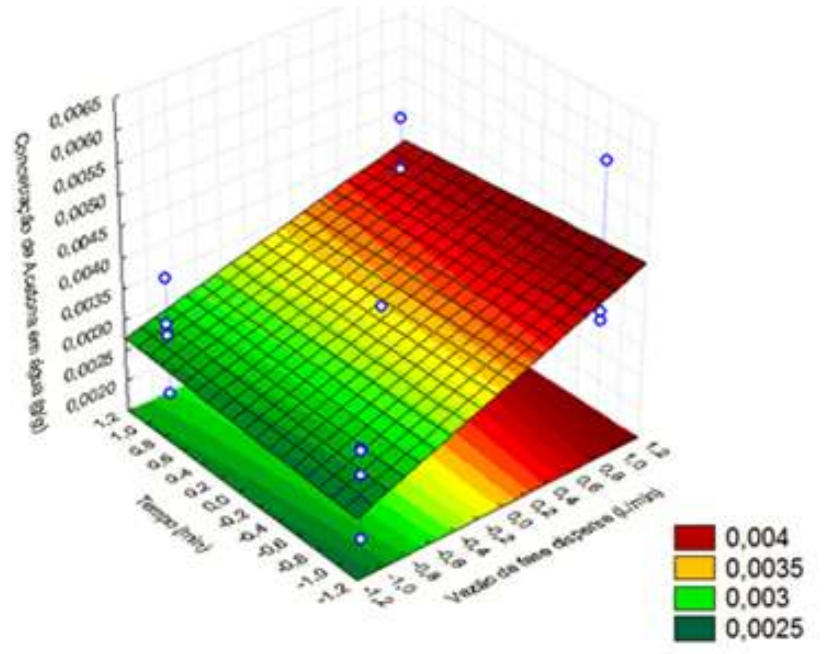

Fonte: Autores.

No gráfico da Figura 11 referido, pode ser feita uma comparação imediata com a Figura 9, já que ambas as vazões das duas fases, contínua e dispersa, estão relacionadas com o tempo, tornando o comportamento das superfícies idênticas. Devendo-se salientar que o tempo só trará melhor resposta para a absorção, quando o a vazão da fase dispersa estiver no nível mais elevado $(+1)$.

Na Figura 12 é mostrada a interação da temperatura e o tempo com a concentração da acetona, sendo mantidas fixas as vazões das duas fases contínua (Qc) e dispersa (Qd).

Figura 12: Superfícies de respostas da variação da concentração da acetona com relação a temperatura (T) e e o tempo (t).

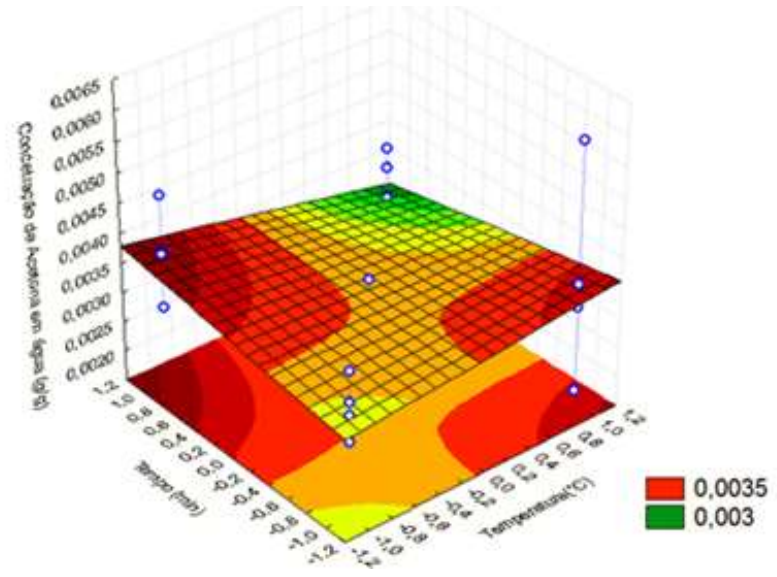

Fonte: Autores. 
No gráfico da Figura 12 observa-se que quando a operação foi realizada com o tempo e a temperatura em seus altos níveis (+1) a resposta para a concentração de acetona em água não apresentou níveis elevados mostrando inclusive uma interação negativa, como já tinha sido mostrado na Tabela 2. Ou seja, quaisquer combinações das variáveis independentes Temperatura e o tempo não se apresenta favorável para o aumente do da concentração na amostra.

Segundo Calado \& Montgomery (2003), um aspecto importante sobre as superfícies de resposta e as curvas de níveis é que quando as linhas que compõem esses gráficos não apresentam curvaturas, pode-se concluir que não há efeito de interação entre as variáveis escolhidas para os eixos. Assim, no caso do presente trabalho, três dos seis gráficos apresentados contém curvaturas acentuadas, sendo consideradas relevantes para o estudo.

\subsection{Análise Dimensional}

A análise dimensional do presente trabalho teve por objetivo a determinação de uma equação empírica em função de grupos adimensionais conhecidos envolvendo a para a queda de pressão (DP), os parâmetros operacionais e de geometria utilizados e as propriedades físicas do sistema. Logo, de acordo com o método, a Queda de Pressão deve ser uma função dos parâmetros listados na função mostrada a seguir. parâmetros geométricos, operacionais e das propriedades físicas do sistema. Ou seja,

$\Delta \mathrm{P}=\mathrm{F}(\mathrm{Qd}, \mathrm{Qc}, \mathrm{Z}, \mathrm{D}, \mathrm{g}, \mu \mathrm{c}, \mu \mathrm{d}, \rho \mathrm{d} \rho \mathrm{c})$

que, utilizando-se a análise dimensional, forma a seguinte função

$$
\frac{\Delta P}{\rho_{d .} \cdot u_{d}^{2}}=\mathrm{G}\left(\frac{u c}{u d}, \frac{\mu_{c}}{\mu_{d}}, \frac{\rho_{c}}{\rho_{d .}}, \frac{u d \cdot g}{\rho d \cdot u_{d}^{3}}, \frac{u d \rho_{d} z}{\mu_{d}}, \frac{u d \rho_{d D}}{\mu_{d}}\right)
$$

Com os dados experimentais obtidos, utilizando a regressão linear multivariável, eliminando-se os grupos que apresentaram constantes com valores reduzidos, foi obtida então a equação 6 descrita a seguir

$$
\frac{\Delta P}{\rho_{c} \cdot u_{c}^{2}}=4,1105 \cdot\left(\frac{u_{d}}{u_{c}}\right)^{0,8317} \cdot\left(\frac{\mu_{c} \cdot g}{\rho_{c} \cdot u_{c}^{3}}\right)^{0,891} \cdot\left(\frac{R e_{d}}{R e_{c}}\right)^{0,3195}
$$

Onde $u_{d}$ e $u_{c}$ são as velocidades superficiais das fases, leve e pesada, e Eu e Re, representam os números adimensionais, de Euler e de Reynolds, respectivamente, ou seja,

$$
E u=\frac{\Delta P}{\rho_{c .} u_{c}^{2}} ; \quad R e_{d}=\frac{u d \cdot D \cdot \rho_{d}}{\mu_{d}} \quad \text { e } \quad R e_{c}=\frac{u c D \rho_{c}}{\mu_{c}}
$$

sendo $\mathrm{Re}_{\mathrm{d}}$ para a fase dispersa e $\mathrm{Re}_{\mathrm{c}}$ para a fase contínua.

A Figura 13 mostra uma comparação entre valores experimentais do número de Euler obtidas com os dados experimentais e os valores calculados através da Equação 6. 
Research, Society and Development, v. 10, n. 10, e76101018592, 2021

(CC BY 4.0) | ISSN 2525-3409 | DOI: http://dx.doi.org/10.33448/rsd-v10i10.18592

Figura 13: Comparação entre os valores do Número de Euler Experimentais e Calculados.

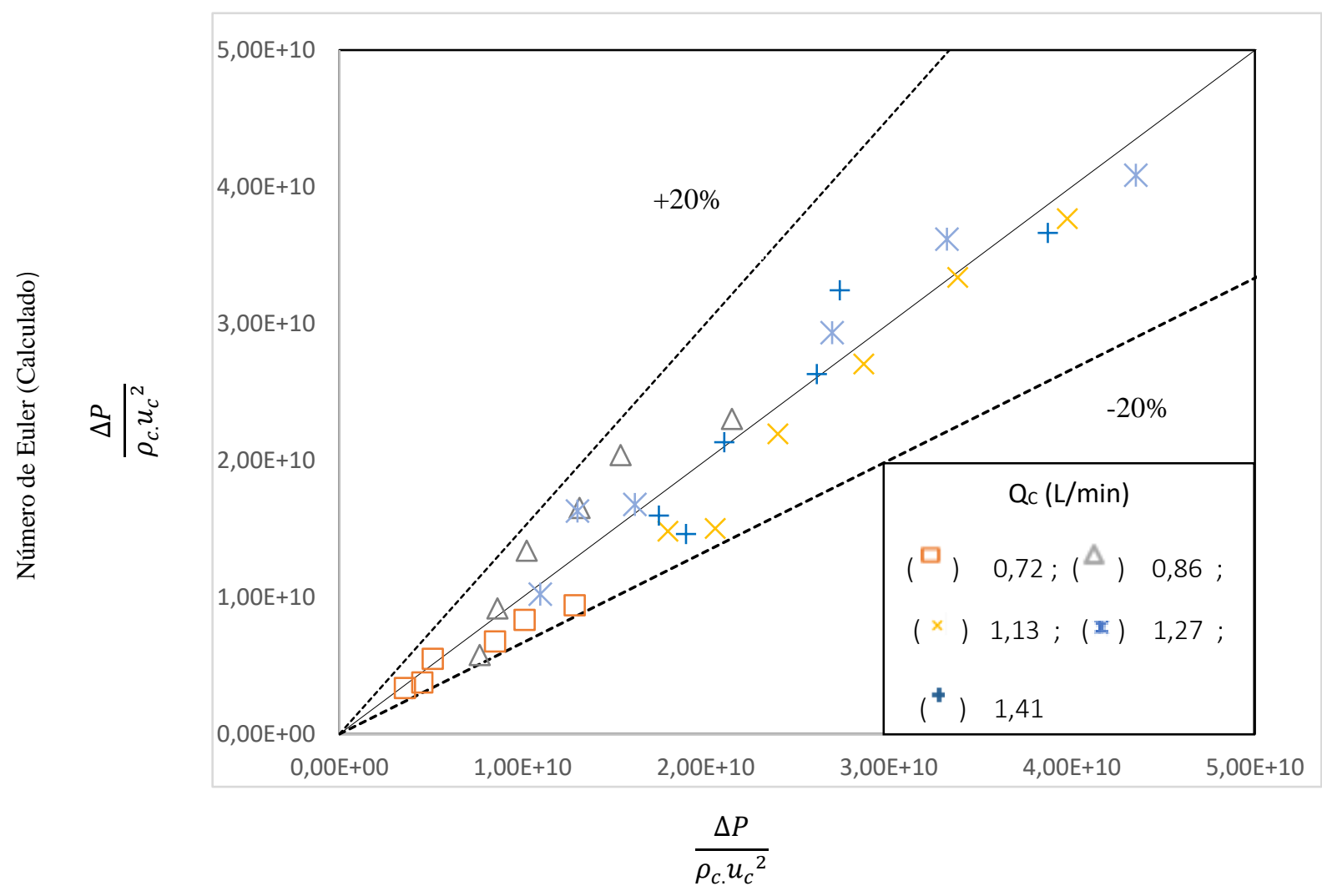

Número de Euler (Experimental)

Fonte: Autores.

De uma forma geral, pode se perceber que a Equação 6 descreve bem os experimentos realizados. já que o erro médio obtido quando relacionados os dois valores, este foi de $13,6 \%$.

Comparando a equação empírica obtida com as descritas na literatura, a que mais se aproximou foi a publicada por Prahl (1970), apresentando erro médio de 28,6\% quando implementado os dados experimentais em sua equação, que desenvolveu uma equação para todos os tipos de recheios sendo limitada para um faixa de 80 e $170 \mathrm{mmH} \mathrm{m}_{2} \mathrm{O} / \mathrm{m} \mathrm{de} \mathrm{recheio}$ de perda de carga, e para valores de parâmetros de fluxo, X, entre 0 e 1,0, range respeitado no procedimento experimental. Analisando o resultado, houve uma redução de $15 \%$ entre as duas equações citadas. A equação desenvolvida por Niranjan (1983), que não utiliza o fator de caracterização dos recheios, obteve um erro médio de 30\%, ou seja, bem superior ao da equação aqui desenvolvida.

\section{Conclusões}

Diante dos dados apresentados no presente trabalho pode-se concluir que vários aspectos foram determinados visando a melhor hidrodinâmica da coluna e, consequentemente, a maior eficiência na operação da absorvedora. Primeiramente foi constatada tanto a forte influência das duas vazões de alimentação na concentração de acetona na saída, 
como também a pouca influência dos outros parâmetros como Temperatura, tempo de saturação, etc. Além disso, duas equações de modelagem foram obtidas com sucesso, sendo a primeira para a concentração de acetona na saída, enquanto a segunda para a Queda de Pressão, através dos valores do número de Euler (Eu). Com isso, conclui-se que com as informações obtidas no presente trabalho progressos significativos foram obtidos, que poderão posteriormente vir a ser utilizados e tornar cada vez mais eficiente a operação da absorção em colunas de recheio

Após a realização do estudo de avaliação dessa separação acetona-água na coluna de recheio, torna-se possível a utilização do aparato experimental para a realização de e de outros estudos hidrodinâmicos, variando a altura do recheio da coluna, como o tipo de recheio, suas características, bem como suas dimensões; interessante também realizar estudos de holdup, simulações do sistema e procedimentos com outros tipos de substâncias visando analisar a variabilidade dos dados junto a essa mudança.

\section{Lista de Símbolos}

G água - Vazão de água (L/min)

G ar - Vazão de ar (L/min)

$\mathrm{U}$ ar - Fluxo molar de ar $\left(\mathrm{kmol} / \mathrm{m}^{2} . \mathrm{s}\right)$

$\Delta \mathrm{P}$ - Queda de Pressão (Pa)

$\mathrm{Z}$ - Comprimento da coluna (m)

\section{Agradecimentos}

Este trabalho teve apoio da CAPES / Brasil para obtenção do título de Mestre de João Carlos Ferreira Lima.

\section{Referências}

Amatuzi,D.R. (1999). Transferencia de Massa Gas-Liquido, 1 Em Leitos Centrifugos - Dissertação de Mestrado - Universidade Estadual de Campinas Faculdade De Engenharia Química. Campinas-SP.

Bernardes, A. P. D. \& Góis, L. M. N. (2020). Evaluation of efficiency and mass transfer in a pulsed packed extraction column. Research, Society and Development,9(2), e132922185. 10.33448/rsd-v9i2.2185.

Barros Neto, B., Scarmino, I.S., Bruns, R. E. (2010).Como fazer experimentos: pesquisa e desenvolvimento na ciência e na indústria. 4 ed. Porto Alegre: Bookman.

Billet, R. \& Mackowiak, J. J. (1984). Fette Seifen Anstrichminel. 86, 342-358.

Cajueiro, R. L. P. (2013). Manual para elaboração de trabalhos acadêmicos: guia prático do estudante/Roberta Liana Pimentel Cajueiro. 2. ed. Petrópolis, RJ.

Calado V. \& Montgomery,D.C.(2003). Planejamento e Experimentos usando o Statistica. e-papers - Rio de Janeiro.

Caldas, J. N.; Lacerda, A. I. (2003). Internos de torres: pratos \& recheios. Rio de Janeiro: EdUERJ, 466 p. ISBN 8575110608.

Dharma, S.; Masjuki, H. H.; Ong, H. C.; Sebayang, A. H.; Silitonga, A. S.; Kusumo, F.; Mahlia, T. M. I.(2016). Optimization of biodiesel production process for mixed jatropha curcas-ceiba pentandra biodiesel using response surface methodology. Energy convers. Manage, 115, 178-190.

Ergun, S. (1952). Fluid flow through packed columns. Chem. Eng. Prog. 48.

Graff, K., Oil \& Gas Journal (1985), May.

Heyouni, A., Roustan, M.; Do-Quang, Z. (2002) Hydrodynamics and mass transfer in gas-liquid flow through static mixers. Chemical Engineering Science, v. 57 , p. $3325-3333$.

Hoffmann R., Terra, L.M., Junior, R. J. S. M. (2011). Validação, Pelo Método Estatístico, da Produção e Alcance da Graduação de Etanol (Álcool Etílico Hidratado) Carburante, Sob o Enfoque das Microdestilarias em Processo de Batelada - ISSN 2178-3659. 
Research, Society and Development, v. 10, n. 10, e76101018592, 2021

(CC BY 4.0) | ISSN 2525-3409 | DOI: http://dx.doi.org/10.33448/rsd-v10i10.18592

Koche, J. C. (2011). Fundamentos de metodologia científica. Petrópolis: Ed Vozes. De http://www.brunovivas.com/wpcontent/uploads/sites/10/2018/07/K\%C3\%B6che-Jos\%C3\%A9-Carlos0D0AFundamentos-de-metodologiacient\%C3\%ADfica-_-teoria-da0D0Aci\%C3\%AAncia-e-inicia\%C3\%A7\%C3\%A30-\%C3\%A0-pesquisa.pdf.

Leva, M. (1953). Tower Packings and Packed tower Design. 2a ed. Akron, Ohio: The United States Stoneware Copany.

Lima, J. C. F.; Costa, J. C.; Matteddi, S.; Góis, L. M. N . (2021) Inundação e carga em coluna de absorção recheada . Research, Society and Development, v. 10, n. 3, e29410312369 DOI: https://doi.org/10.33448/rsd-v10i3.12369.

Nasser, J. R.\& Taqueda M. E. S. (2008) Replacement of Trays by Packing To Increase the Absorption Capacity of Acetone during Cellulose Acetate Spinning Roberto - Ind. Eng. Chem. Res. 2008, 47, 8376-8383.

Niranjan, K. (1983) Chem. Eng., 90, 67.

Pereira, A. S., Shitsuka D.M., Parreira, F.J. \& Shitsuka, R. (2018). Metodologia da pesquisa científica. [free ebook]. Santa Maria: UAB/NTE/UFSM. https://www.ufsm.br/app/uploads/sites/358/2019/02/Metodologia-da-Pesquisa-Cientifica_final.pdf.

Prahl, W. H. (1970). Liquid Density Distorts Packed Column Correlation. Chemical Engineering, 77 (24), 109-112.

Rodrigues, M. I.\& Iemma, A. F.(2005) Planejamento experimental e otimização de processos: uma estratégia de planejamento sequencial. Campinas, SP. Casa do Pão Editora.

Simonelli, G.; Moraes, C.; Pires, C. A. M.; Santos, L. C. L. (2019).Multivariate Study and Optimization of Biodiesel Production Using Commercial Surfactants. Chem. Ind. Chem. Eng. Q. 25 (2), 183-192.

TreybaL, R E. (1980). Mass-Transfer Operations, Third Edition. McGraw-Hill Book Company, London.

Troendle, James F. (2008). Biometrical Journal, August. Vol.50(4), pp.571-583. 